\title{
Effects of the structure of descriptions on group impression formation
}

\author{
KEITH R. STRANGE, MARK SCHWEI, and RALPH E. GEISELMAN \\ University of California, Los Angeles, California 90024
}

\begin{abstract}
A within-subjects design was used to study the impression formation of groups that were held constant in length of description, but differed in the order of presentation of the desirable and undesirable members and in the number of members. The procedure followed closely that of previous person impression-formation studies (Anderson, 1972). A significant primacy effect was found when one adjective was used to describe each member, but a significant recency effect was found when three adjectives were used to describe each member. It was suggested that if a reasonable amount of information is given for each member of a group, then for a more desirable impression to be formed overall, the most desirable members should be presented last. This suggestion is the opposite of that which has been proposed to maximize the desirability of an individual person.
\end{abstract}

In the formation of impressions of individual persons, studies have reliably demonstrated that the first adjective traits used to describe a person are weighted more heavily in the impression than are adjectives presented later in the list of traits (Anderson, 1973). Several processes have been proposed to account for this primacy effect, but the hypothesis that currently has the most support appears to be the attention-decrement hypothesis (Anderson, 1973; Hendrick \& Constantini, 1970) in conjunction with a differential weighting model. This hypothesis states that as a list of adjectives is presented, attention (and thus cognitive processing and the weight parameter in the overall impression) decreases as a function of input serial position in the absence of any distinct list event to renew attention.

A systematic study of the factors influencing the primacy effect as it concerns the formation of impressions of groups has been lacking. The major purpose of the present experiment was to provide such a systematic study. In the present experiment, the distinctiveness of the stimuli in the adjective trait list was varied by holding the number of adjectives constant and varying the number of members in a group. If attention decreases as a function of input serial position and is then renewed with the presentation of each new group member, the weight parameters of the adjective traits presented later in the description of each group should be higher when the presentation of each group member is a more infrequent event. That is, with fewer members in a group, the weight parameters of adjective traits presented later in the list should increase, possibly producing a recency effect in the subjects' ratings of the group. In a pilot study involving group impression formation, the authors

Requests for reprints may be sent to Ralph E. Geiselman, Department of Psychology, University of California, Los Angeles, California 90024. observed a nonsignificant recency effect in the subjects' ratings of four-member groups with three adjectives per member. A similar study, conducted recently by Lyons, Bjork, and Riskey (Note 1), produced comparable results.

With a large number of members in a group and, consequently, fewer adjectives per group member, the presentation of a new member would be a more frequent event and should be less likely to renew the rater's attention. Thus, the primacy effect seen in previous studies of person impression formation should be obtained.

Another phenomenon of information integration that was investigated involves rating inconsistent information negatively regardless of the relative values of positive and negative aspects of the information. This form of inconsistency discounting has been demonstrated previously in studies of moral judgment (Birnbaum, 1973) and verbal vs. nonverbal demands (Bugental, Kaswan, \& Lowe, 1970). In these studies, when subjects were presented information that contained both positive and negative qualities, they rated the information negatively. To determine whether this phenomenon is evident when impressions of groups are formed, the consistency of information contained in the descriptions of the group members was varied in the present experiment. A lower rating for groups with inconsistent members than for groups with consistent members could be interpreted as evidence for inconsistency discounting in group impression formation.

\section{METHOD}

\section{Subjects}

The subjects were 30 undergraduate volunteers obtained from the introductory psychology course at the University of California at Los Angeles. 


\section{Materials and Apparatus}

The stimulus materials were 24 sets of 12 adjectives selected randomly from Anderson's (1968) lists of adjectives scaled for likableness. Only the high- and low-likability adjective lists were used. Within each group of adjectives, six adjectives were taken from the high lists and six were taken from the low list. The 24 adjective lists were used as descriptions of 24 "stimulus groups" in the experiment.

The number of members in each group was varied by designating each group as having one, two, or three adjectives in the description of each of its members. The order of presentation of the 12 adjectives within each group was varied in four levels (HL, $\mathrm{LH}$, random-homogeneous, and random-heterogeneous). In the HL groups, the first six adjectives were taken from the high list and the last six adjectives were taken from the low list; in the LH groups, the first six adjectives were taken from the low list and the last six from the high list; in the random-homogeneous groups, the adjectives were randomly ordered but each person was described by either all high or all low adjectives; in the random-heterogenous groups, all 12 adjectives were randomly ordered without regard to the number of persons in the group.

Each group was named by a letter of the alphabet, and each group member was designated by a number from 1 to 12 , corresponding to the member's position in the presentation of the group.

The 24 groups were randomly ordered and their descriptions were recorded on tape, listing the group name, and then each member along with the adjectives used to describe each person. Following the presentation of each group, the subjects were asked to rate that group and were allowed $10 \mathrm{sec}$ to make their rating. This sequence was followed for each group until all of the groups had been presented. The mean time for presentation of each member was calculated at 2.6,3.4, and $4.4 \mathrm{sec}$ for the onetwo-, and three-adjective/member groups, respectively.

\section{Procedure}

The subjects were tested in two groups of 15 subjects. All subjects were informed that they would be asked to make ratings of a number of groups with respect to how much they would like that group. In addition, they were told that the group members approximated the subjects' peer group. They were then introduced to a 9-point rating scale (which was printed on their rating sheet). On the scale, 0 denoted a rating of the group as "least likable, favorable, or desirable) and 8 indicated that the group was "most likable, favorable, or desirable." End anchors were given, and the subjects rated four practice groups in order to familiarize themselves with the rating scale. The procedure used here duplicated as closely as possible Anderson's procedures used in previous experiments in person impression formation (Anderson, 1972). Subjects then listened to the tape recording and rated the groups.

\section{Design}

A 3 by 4 by 2 within-subjects design was used, with the factors being number of adjectives per group member (one, two, or three), order of presentation of adjectives (HL, LH, randomhomogeneous, and random-heterogeneous), and replication (there were two instances of each type of group). The dependent variable was the subjects' ratings of each group for likableness.

\section{RESULTS}

The analysis of variance conducted on the group ratings showed that both the main effect of order of presentation of the adjectives and number of adjectives per group member were not significant $[F(3,87)=1.12$ and $F(2,58)=.51$, respectively] . However, the Number

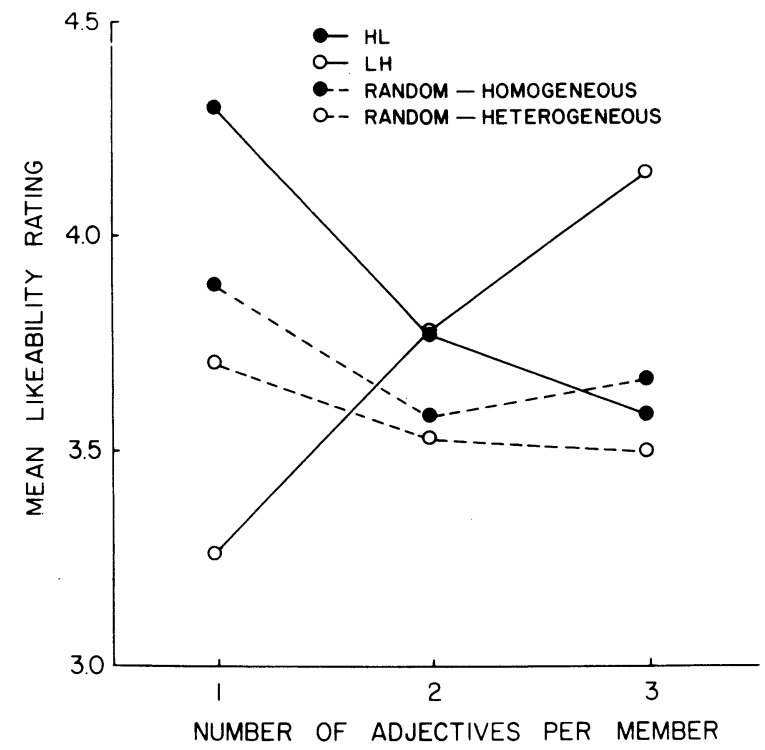

Figure 1. The mean likability ratings for the four types of groups as a function of the number of adjectives per group member.

by Order interaction effect was significant $[F(6,174)=$ 5.71, $\mathrm{MSe}=1.25, \mathrm{p}<.01]$. A Cicchetti test (Cicchetti, 1972) conducted on the interaction showed that with one adjective per member, the mean rating for the HL groups was significantly higher than for the LH groups $(p<.01)$; with three adjectives per member, this relationship was reversed $(\mathrm{P}<.05)$. In addition, with one adjective per member, the mean rating for the HL groups was higher than for the random-heterogeneous groups ( $p<.05)$, and the mean rating for the randomhomogeneous groups was higher than for the LH groups $(p<.05)$. With three adjectives per member, the mean rating for the LH groups was higher than for the random-heterogeneous groups $(p<.05)$.

Inspection of Figure 1 indicates that, for the HL groups, ratings were lower with greater numbers of adjectives per member, but that, in the LH groups, ratings were higher with greater numbers of adjectives per group member. In the random-homogeneous and random-heterogeneous groups, no significant trend was seen, although these ratings were somewhat higher with only one adjective per group member than with two or three adjectives per group member. In addition, the random-homogeneous groups were rated somewhat higher than the random-heterogeneous groups, but this difference was not statistically significant.

\section{DISCUSSION}

The findings of this experiment fall into two main categories -those pertaining to the HL and LH groups and those pertaining to the random groups.

First, when one adjective was used to describe each member of a group, a primacy effect was obtained. That is, HL group 
ratings were higher than LH group ratings. This is consistent with previous findings in person impression formation and suggests that with one adjective given for each person, the impression of the group is formed in a manner that is similar to the way in which the impression of a single person is made. However, when three adjectives were given in the description of each person, the primacy effect was replaced by a recency effect. That is, LH group ratings were higher than $\mathrm{HL}$ group ratings. This is a departure from results of person impression-formation experiments and suggests that the rater is processing the available information differently when the stimulus to be rated is more like a group and less like an individual person.

These results are consistent with the attention-decrement hypothesis, which states that the terminal adjectives are given less weight in the overall impression because they receive less attention and thus are processed less meaningfully by the rater. Here it is proposed that when one adjective was given per member, attention decreased as the list was presented in the absence of any novel list events. However, when three adjectives were presented for each group member, the presentation of each group member became a more distinct or novel list event due to either a greater separation of the members in time or to a greater separation of the members in the stimulus list of adjectives. This may renew the rater's attention, and may induce the rater to give more weight to adjectives presented later in the list. With two adjectives per member, possibly an intermediate effect is observed and the HL and LH curves cross due to a combination of the attentional factors that influence both the initial and terminal portions of the list. The actual point of crossover is not important here, and is probably a relative function of the context of the experiment and the types of groups used.

Although the difference between the random-homogeneous group ratings and random-heterogeneous ratings was not significant, the fact that the mean random-homogeneous ratings were consistently higher than the mean random-heterogeneous ratings is at least suggestive of a tendency for subjects to rate inconsistent information negatively rather than positively. An abstraction of the concept of inconsistency discounting can also explain the finding that when two adjectives were presented per group member, the ratings for both types of random groups were somewhat lower than those for the HL and LH groups. If the groups are seen as clusters of adjective traits presented in the sequence, then in the random groups, any given cluster is more likely to contain low adjective traits than that same sequential cluster in a HL or $\mathrm{LH}$ group due to the random interspersing of either negative persons or negative adjective traits. Thus, any given cluster is more likely to be rated negatively, and the group is more likely to be rated negatively as a result. It is interesting to note that with three adjectives per member, having a mixture of inconsistent members or low and high members gives no better impression than having all low persons at the end of the list.

The strength of this study lies in the fact that, in one condition of the experiment, results were obtained that are consistent with those found in previous studies of person impression formation, while evidence for other types of information integration was found when the structure of the list of adjectives was changed. The latter results suggest that if enough information is given for each member of a group, the presentation of a new member may serve to renew the rater's attention. Hence, for a favorable impression of a group to be formed, it is probably the case that the more desirable members should be presented last.

\section{REFERENCE NOTE}

1. Lyons, G., Bjork, R. A., \& Riskey, D. Group impression formation. Manuscript in preparation, University of California at Los Angeles, 1978.

\section{REFERENCES}

ANDERSON, N. H. Likeableness ratings of 555 personality-trait words. Journal of Personality and Social Psychology, 1968, 9. 272-279.

ANDERSON, N. H. Information integration theory: A brief survey (Report 24). La Jolla, Calif: Center for Human Information Processing, April 1972.

Anderson, N. H. Serial position curves in impression formation. Journal of Experimental Psychology, 1973, 97, 8-12.

Birnbaum, M. H. Morality judgment: Test of an averaging model with differential weights. Journal of Experimental Psychology, 1973, 99, 395-399.

Bugental, D. E., Kaswan, J. W., \& Lowe, L. R. Perception of contradictory meanings conveyed by verbal and nonverbal demands. Journal of Experimental Psychology, 1970, 16, 647-655.

Ciccherti, D. V. Extension of multiple-range tests of interaction tables in the analysis of variance: A rapid approximate solution. Psychological Bulletin, 1972, 77, 405-408.

Hendrick, C.. \& Constantini, A. F. Effects of varying trait inconsistency and response requirements on the primacy effect in impression formation. Journal of Personality and Social Psychology, 1970, 15, 158-164.

(Received for publication May 30, 1978.) 